\title{
Design of Portable LED Stage Lighting Control System
}

\author{
Huiqin Wang \\ School of Information Engineering \\ Communication University of China \\ Beijing,China \\ hqwang@cuc.edu.cn
}

\begin{abstract}
Compared with the conventional dimming system of LED stage light which uses the heavy light operating console to adjust the brightness of stage light, a portable LED stage lighting control system is designed in this paper. In this system a adjust handle is introduced to adjust the brightness of the LED stage light instead of the operating console, accordingly the novel dimming system is portable and easy to carry compared with the conventional one. The key technologies of the system such as high power LED driving circuit ,LED dimming and the heat dissipation of LED are analyzed here in detail. Simulation and experiment results have shown the effectiveness of the proposed system.
\end{abstract}

Keywords—high power LED; LED driving circuit; PWM; LED thermal analysis

\section{INTRODUCTION}

White light emitting diode (LED) with high brightness has attracted a lot of attention from both industry and academic for its high efficiency, ease to drive, environmental friendliness, and long lifespan. They become possible applications to replace the incandescent bulbs and fluorescent lamps in residential, industrial as well as commercial performance, stage lighting. The portable LED stage lighting control system is designed in this paper to meet the demand of variety, realtime and portability of stage light dimming.

Compared with the traditional dimming system which is composed of light operating console, dimming controller and other auxiliary components, the control system proposed here use a dimming handle to take place of the heavy operating console to constitute a more portable LED stage lighting dimming system. Fig. 1 shows the structure of the proposed dimming system.

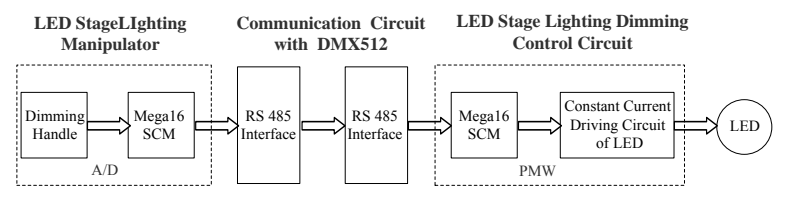

Fig.1. Diagram of control system of LED stage lighting

\author{
Zhen Li \\ School of Information Engineering \\ Communication University of China \\ Beijing,China \\ lizhen@cuc.edu.cn
}

The overall system shown in Fig.1 is composed of three parts, LED stage lighting controller, LED stage lighting dimming circuit and the communication circuit with DMX512 protocol. LED stage lighting controller is the transmit part of the whole system. With pulling the dimming handle, the output voltage of the handle is changing consequently, then the voltage is converted into a 10 - bits digital signal by the analog / digital convert unit of single-chip microcomputer (SCM). Subsequently, the digital signal is packaged according to the DMX512 data transmission protocol and sent to the SCM's output port. Through the first part, the signal converts for analog into data segment meeting DMX512 protocol format. Then using two RS485 serial interfaces, the data is sent to the third part-- LED stage lighting dimming circuit. The SCM of the dimming circuit generates a pulse width modulation(PWM) control signal to drive constant current source circuit according to the dimming signal received. Finally, the constant current driving system dims the LED lights based on the PWM signal. Because the luminance is mainly affected by LED driving current, so the selection of LED driving mode will directly influence the effect of light dimming. Meanwhile due to the total power of LED stage light is very high, thus the thermal problem of LED can't be omitted, so the technology about heat dissipation is also involved in the paper.

Altogether the major technologies of this high power LED stage light dimming system including LED lamp drive circuit selection, LED lamp technology system of PWM modulation technology and heat dissipation technology, each of these technologies are discussed in detail as follows. The driving circuit of high power LED stage light is proposed in Section II. The DMX 512 data transmission protocol is described in Section III. The thermal analysis of LED stage light is studied in Section IV. Conclusions and experiment results are shown in Section V.

\section{THE DRIVING CIRCUIT OF HIGH POWER LED STAGE LIGHT}

\section{A. Selecting Proposed LED Drive Circuit}

According to the electrical characteristic of high-power LED array, there are two major driving methods, constant 
voltage driving method and constant current driving method .The former has the advantages of simple circuit, low cost. But when using this method, with the LED junction temperature rising, LED forward voltage-drop will decrease, furthermore under the same power, the positive current increases, which in turn leads to the rise of the LED junction temperature again, at last LED chip will damage. As to the latter, using this method can make the LED light stability; avoid the change of the positive current caused by the vibration of LED positive voltage[1][2]. Through setting the optimal working current according to the characteristics of LED chips, can prolong the working life of the LED, and ensure the luminous efficiency. So most of the high power LED driver nowadays, take this driving mode. As to choice of the high power LED stage light designed here, we use the constant current drive method. Fig.2. shows the proposed constant current driving circuit .

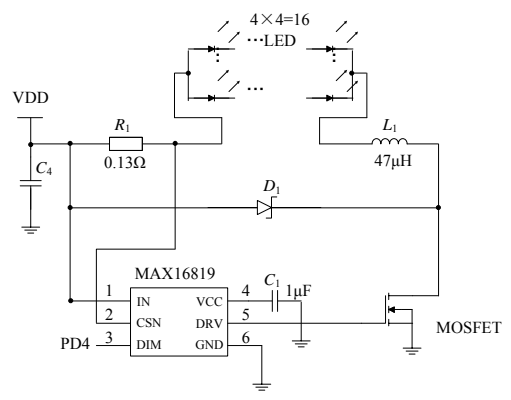

Fig.2. The constant current driving circuit on chip MAX16819

The high-power LED stage light constant current drive circuit designed here is basically constructed by one current detection resistor $R_{1}$, one inductor $L_{1}$, two capacitors $C_{1}$ and $C_{4}$,one MOSFET, one freewheeling diode $D_{1}$ which can provides a continuing loop for the current flowing through the inductor $L_{1}$ during the MOSFET closes, and step-down constant-current high-brightness LED (HB LED) driver -MAX16819. The chip is a buck converter. It operates from a $4.5 \mathrm{~V}$ to $28 \mathrm{~V}$ input voltage range and features a $5 \mathrm{~V} / 10 \mathrm{~mA}$ onboard regulator. The output current is regulated by high side current sensing resistor $R_{1}$, adjustable pulse brightness achieve wide range. The hysteretic on-time controller and an external resistor allow the converter output voltage to adjust as needed to deliver a constant current to a series-parallel connected LED arrays. This chip used here to provide an adjustable constant forward current four strings of four series connected 5W LEDs, the following describes the driver circuit in detail [3].

In this circuit, a $12 \mathrm{~V}$ DC voltage signal, VDD is created as the LED's current as well as the supply voltage of MAX16819. As to the LED current it is programmable using resistor $R_{1}$. The MAX16819 regulates the LED output current using an input comparator. As the current through the inductor $L_{1}$ ramps up and the voltage across $R_{1}$ reaches the upper threshold, the voltage at DRV goes low, turning off the external MOSFET. The MOSFET turns on again when the inductor current ramps down through the freewheeling diode $D_{1}$ until the voltage across $R_{1}$ equals the lower threshold.

\section{B.Maintain PWM Dimming Mode}

In high power LED stage light driving circuit, PWM mode is used in this paper, in which the frequency of the control pulse is constant and the average current is controlled by changing the pulse width. The PWM signal output through the PD4 port of the SCM is sent into DIM. A logic level below $0.6 \mathrm{~V}$ at DIM forces DRV output low, turning off the external MOSFET. When the logic level at DIM is more than $2.8 \mathrm{~V}$, the MOSFET turns on. With the MOSFET being on, the freewheeling diode- $D_{1}$ cuts off, VDD began to power supply the inductor $L_{1}$, the high current ripples to the peak value linearly in the loop of VDD- $R_{1}$-ILEDs- $L_{1}$-MOSFET-GND. When the MOSFET is off, $D_{1}$ turns on, providing a continuous path for the inductor current, then the high current circulates through $R_{1}$-ILEDs- $L_{1}-D_{1}$. Its value ripple down for the peak value.

The dedicated PWM input DIM enables a wide range of pulsed dimming which is available for the need of the brightness variety for high-power LED stage light. The dimming signal obtained from the data package which sent from stage lighting manipulate system is transmitted to DIM port shown on Fig. 2 through the output of PD4 of the stage lighting controller. When the dimming circuit shown as Fig.2 With the dimming signal changing, the waveform of the PD4 changes consequently which results in the varies of duty circle, finally the brightness of LED lights change.Fig.3 shows the PWM waveform of DIM, different duty circles control different brightness of stage lighting.

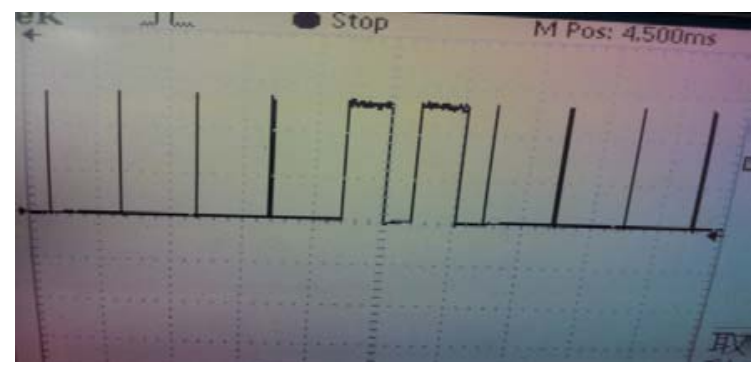

Fig.3. the PWM waveform of port DIM

\section{DMX-512 PROTOCOL}

DMX512 Protocol specified by the Technology Association of America Theater in 1987, it is an entertainment industry standard based on RS485, which is mainly used to control stage lighting. It covers electrical characteristics (based on EIA/TIA-485 standard), data format, data protocol, and connector type. Meanwhile, it is an Asynchronous Serial Digital Data Transmission Standard for controlling lighting equipments and accessories [4].

DMX512 controller transmits data package to dim the stage lighting. The data transmitted shall be in asynchronous serial format and must meet the format and the timing of DMX512 protocol. That is fixed at one start bit, eight data bits, 
two stop bits and no parity. Prior to the first level transmitted, a RESET signal shall be transmitted followed by a NULL START code. Valid dimmer levels shall be to 255 decimal (00 to FF hexadecimal) representing dimmer control input levels of OFF to FULL in a linear relationship. Fig.4 shows the timing diagram of the data package in detail [5]

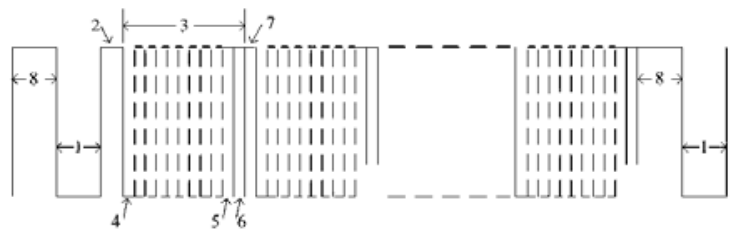

Fig.4. Timing Diagram of the Data package

In this design, the RESET signal (Fig 4. \#1) shall consist of a BREAK lasting $120 \mu \mathrm{s}$. A BREAK shall be defined as a high-to-low transition. All dimmers and other receiving devices shall interpret any such BREAK as a terminator for any pending transmission/data packet and its end as the start of the MARK AFTER BREAK and START code sequence at the beginning of the next packet. The duration of the MARK separating the RESET/BREAK and the START code (Fig 4. \#2) lasts $12 \mu \mathrm{s}$. Designation \#3--a data segment identifies subsequent data as sequential 8-bit dimmer level information. Designation \#4 is the start bit of data segment. Designation\#5 and \#6 are the stop bit of the segment. Designation \#7 is the idle period between segments varying from $0 \mu$ sand $1 \mathrm{~s}$. The time between any two frames of a data packet (Fig 4.Designation \#8) may vary between $0 \mu$ s and $1 \mathrm{~s}$. The line must remain in a "marking" state during any such idle period greater than $0 \mu \mathrm{s}$. A receiver must be capable of accepting a data packet having no idle time $(0 \mu \mathrm{s})$ between any of it frames. Every data packet transmitted on the data link, regardless of START code or length, must begin with a RESET, MARK AFTER BREAK, and START code sequence as defined above.

\section{HEAT DISSIPATION TEHCNOLOGY OF LED LIGHT}

\section{A. The structure of LED heat sink}

For high power LED stage light, thermal management is one of critical factors for their high performance. In general, most of the electronic power of high power LED is converted into heat, which greatly reduces the chips' luminosity. In addition, the high junction temperature of LED chips in the stage light will shift the peak wavelength, which will change the color of light. Narendran[6] have experimentally demonstrated that the life of LEDs decreases with the increase of the junction temperature in an exponential manner.

For high-power LED stage light of complex shape, as air flow is obstructed by light shell, it is difficult to dissipate heat through nature convection. Fin heat pipe with fan is select here to solve the problem[7]. Heat is conducted through heat pipe to unobstructed space for air flow, and then dissipated through nature convection on fins. Fig. 4 illustrates the arrangement of the LED thermal module. The configuration of the heat sink assembly is a heat pipe with fins array. The base plate is mounted to the LED panel that conducts the dissipated heat. The fins are combined with the base plate for natural convection. The radium of base plate is $53 \mathrm{~mm}$, the thickness of it is $6 \mathrm{~mm}$. Each fin size is $50 \mathrm{~mm} * 60 \mathrm{~mm}$, the height is 60 $\mathrm{mm}$, and the thickness is $2 \mathrm{~mm}$. As to heat pipe, its' section area is $80 \mathrm{~mm}^{2}$, and its' length is $60 \mathrm{~mm}$. Note that the LED chips are mounted to the pane. Each LED chip is prescribed with $5 \mathrm{~W}$ power input having an efficiency of $20 \%$. Its size is $3.4 \mathrm{~mm} * 3.4 \mathrm{~mm}$. The radium of pane is $14 \mathrm{~mm}$. There is a fan at the bottom of the sink, when the temperature of the light is more the $25^{\circ} \mathrm{C}$, the fan will work.

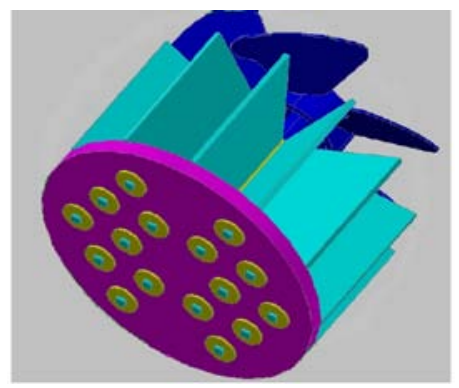

Fig.5 The structure of LED heat sink

\section{B. Simulation model}

In this research, ANASYS software is used for the simulations, and efforts are focused upon made the temperature of LED stage lighting composed of 16 LEDs and its heat sink module. The simulations are performed at an ambient temperature of $25^{\circ} \mathrm{C}$, the LEDs are assumed as the concentrated individual heat sources. The simulations are conducted under natural convection and steady laminar flow condition.

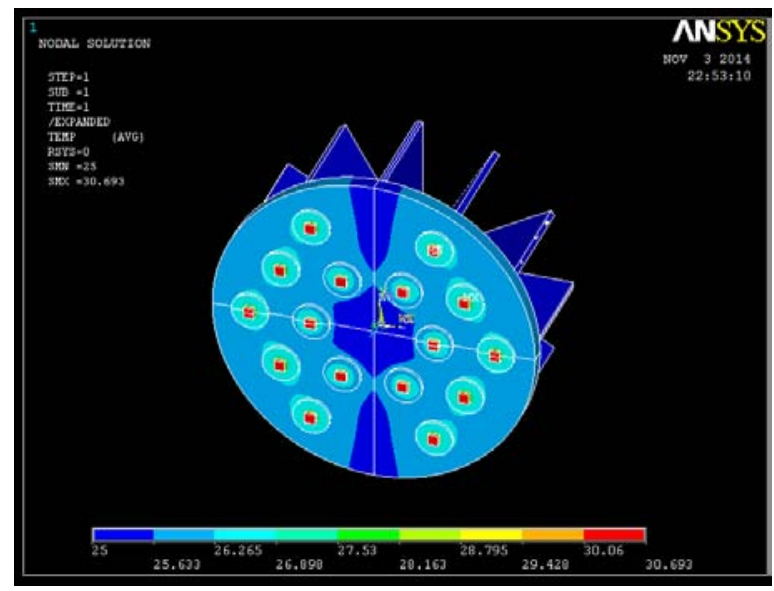

Fig.6 The result of thermal distribution of stage light with ANSYS software

Fig. 6 shows the thermal distribution of the LED thermal module. It represents the highest and lowest temperature of LED lighting. That result explores thermal distribution of the light is non-uniform, the end of fins are quite lower than the region nearby the LED lighting in. The highest temperature is 
$30.693^{\circ} \mathrm{C}$ at the each LED, the lowest temperature is $25^{\circ} \mathrm{C}$. The farther from the LEDs, the lower of the temperature are .The place of LED concentrated ,the thermal coupling effect is intensive, so the temperature is high. As to the center of the light, there is no LED chip and the effective of the thermal soured is small ,so its temperature is low. Altogether, the temperature distribution is within the LED normal work area,so the proposed heat sink is feasible.

\section{RESULTS AND CONCLUSION}

From section II to section IV, we introduced the key technologies of the portable LED stage lighting dimming system. The whole system shows as Fig.7.

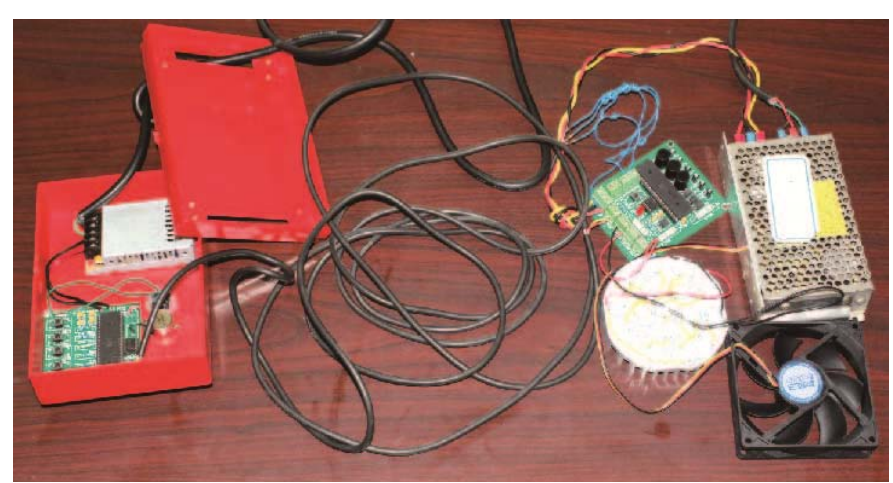

Fig.7. The portable LED stage lighting dimming system

The red box in Fig.7 is the LED stage light dimming system , and the green one is the light manipulator. Pulling the handle of the manipulator, the brightness of the light will change followed under the work of stage lighting dimming system.

To prove the heat dissipation effective of the heat sink use here, the temperature distributions of the aluminum base and fins of the stage light were measured by thermocouples in the experiments. The ambient temperature was about $25{ }^{\circ} \mathrm{C}$, several thermocouples were placed at different positions of the aluminum base and fins. The temperature data obtained by the thermocouples was transferred to the data acquisition system and displayed on TP4 temperature recorder[8].
From the experiment, the highest temperature is $32.5^{\circ} \mathrm{C}$ at LED chips , and the lowest temperature is $25.8^{\circ} \mathrm{C}$, and the temperature increased as time extended, initially, the fin temperature was nearly the same as the room temperature. After the lamp was activated, its temperature increased. Several hours later, it reached steady situation and the maximum temperature remained to be stable to be nearly $32^{\circ} \mathrm{C}$.Compared with the simulation result, it is clear that the modeling results are close to the experimental ones the temperature difference achieved by the two methods was below $2{ }^{\circ} \mathrm{C}$. The comparison proves the feasibility of the structure of heat sink introduced here.

Altogether, in this paper the key technologies such as LED driving circuit, LED thermal analysis etc of portable dimming system for high power LED stage lighting are elaborated. The thermal resistance network model for stage lighting was proposed and was validated by experiments. Compared with the conventional dimming system ,the system designed here is easier to carry, have more convenience to use.

\section{REFERENCES}

[1] Yuequan. Hu, and Milan. M. Jovanovi'c. "LED Driver With Self-Adaptive Drive Voltage Power Electronics," IEEE Transactions, Vol. 23: pp.31163125. Nov. 2008.

[2] Mohammad Kamil. Switch Mode Power Supply (SMPS) Topologies (Part I). Microchip Technology Inc, 2007.

[3] Zhang Can, Qin Huibin, and Qu Liyang," LED Dimming System of Wireless Remote Controller, " IEEE JOURNAL ON SELECTED AREAS IN COMMUNICATIONS, vol. 28, No. 3, APRIL 2010

[4] Wei. Jiang ,Yujian. Jiang, Hui.Ren, "Analysis and Prospect of Control System for StageLighting," 2010 3rd International Congress on Image and Signal Processing, pp.3923-3929,2010.

[5] E1.11-2004 USITT_DMX521A, pp.15-20,2004.

[6] Narendran, N., and Gu, Y. M. "Life of LED-Based white light sources, "IEEE Journal of DisplayTechnology, vol.1, pp.167-171,2005.

[7] Song-Bor Chiang, "The Thermal Analysis of Sequential LED Driven Mode,'International Microsystems,Packaging,Assembly and Circuit Technology conference ,pp.203-205,2009.

[8] Xiaobing Luo, Wei Xiong, Sheng Liu," A Simplified Thermal Resistance Network Model for High Power LED Street Lamp," 2008 International Conference on Electronic Packaging Technology \& High Density Packaging,2008. 